\title{
On Syzygy Modules over Laurent Polynomial Rings
}

\author{
Morou Amidou (D) $^{1}$ and Ousmane Moussa Tessa ${ }^{2}$ \\ ${ }^{1}$ Institut de Recherche sur l'Enseignement des Mathématiques, Abdou Moumouni University, P.O. Box 10896, Niamey, Niger \\ ${ }^{2}$ Department of Mathematics and Computer Science, Abdou Moumouni University, P.O. Box 10662, Niamey, Niger
}

Correspondence should be addressed to Morou Amidou; moorou_a@yahoo.fr

Received 23 December 2019; Accepted 21 March 2020; Published 1 May 2020

Academic Editor: Vladimir V. Mityushev

Copyright (c) 2020 Morou Amidou and Ousmane Moussa Tessa. This is an open access article distributed under the Creative Commons Attribution License, which permits unrestricted use, distribution, and reproduction in any medium, provided the original work is properly cited.

\begin{abstract}
In this paper, we present a dynamical method for computing the syzygy module of multivariate Laurent polynomials with coefficients in a Dedekind ring (with zero divisors) by reducing the computation over Laurent polynomial rings to calculations over a polynomial ring via an appropriate isomorphism.
\end{abstract}

\section{Introduction}

Our goal is to give a dynamical method for computing a finite basis for the syzygy module of finitely many multivariate Laurent polynomials with coefficients in a Dedekind ring R. More precisely, given nonzero polynomials $f_{1}$, $\ldots, f_{s} \in \mathbf{R}\left[x_{1}^{ \pm 1}, \ldots, x_{n}^{ \pm 1}\right]$, we will compute $s_{1}, \ldots, s_{t} \in$ $\mathbf{R}\left[x_{1}^{ \pm 1}, \ldots, x_{n}^{ \pm 1}\right]^{s \times 1}$ generating the syzygy module $\operatorname{Syz}\left(f_{1}, \ldots, f_{s}\right):=\left\{{ }^{\mathrm{t}}\left(w_{1}, \ldots, w_{s}\right) \in \mathbf{R}\left[x_{1}^{ \pm 1}, \ldots, x_{n}^{ \pm 1}\right]^{s \times 1}\right.$

such that $\left.w_{1} f_{1}+\cdots+w_{s} f_{s}=0\right\}$. The technique consists in reducing the computation over the Laurent polynomial ring $\mathbf{R}\left[x_{1}^{ \pm 1}, \ldots, x_{n}^{ \pm 1}\right]$ to a problem over a polynomial ring $\mathbf{R}\left[x_{1}, \ldots, x_{n}, y\right]$ via an appropriate isomorphism. One advantage of this indirect approach is that we can use techniques over polynomial rings more efficient than the corresponding methods in Laurent polynomial rings. Such kind of algorithm can be used in signal processing for the computation of the inverse FIR filter of a given multidimensional FIR filter. Our approach is inspired by the theory developed in the papers [1-13] and outlined in the next section.

\section{Computing Dynamically a Basis for Syzygies of Polynomials over Dedekind Rings}

Let $S$ be a multiplicative subset of a ring $\mathbf{R}$; then, the localization of $\mathbf{R}$ at $S$ is the $\operatorname{ring} S^{-1} \mathbf{R}=\{(x / s), x \in \mathbf{R}, s \in S\}$ and the elements of $S$ are forced to be invertible. If $x \in \mathbf{R}$, the localization of $\mathbf{R}$ at the multiplicative subset $\mathscr{M}(x)$ generated by $x$ will be denoted by $\mathbf{R}_{x}$. Moreover, by induction, for each $x_{1}, \ldots, x_{k} \in \mathbf{R}$, we define $\mathbf{R}_{x_{1} \cdot x_{2} \ldots \ldots x_{k}}:=\left(\mathbf{R}_{x_{1}, x_{2} \ldots \ldots x_{k-1}}\right)_{x_{k}}$.

Now, let $\mathbf{R}$ be a Dedekind ring and consider $f_{1}, \ldots, f_{s} \in \mathbf{R}\left[x_{1}, \ldots, x_{n}\right] \backslash\{0\}$. First of all, we need to present a dynamical process [6] for computing a basis for $\operatorname{Syz}\left(f_{1}, \ldots, f_{s}\right)$. This method works like the case where the basic ring is a Noetherian valuation ring [11]. The Noetherian hypothesis is added so that a dynamical Gröbner basis $G=\left\{\left(S_{1}, G_{1}\right), \ldots,\left(S_{k}, G_{k}\right)\right\}$ for the ideal $\left\langle f_{1}, \ldots, f_{s}\right\rangle$ of $\mathbf{R}\left[x_{1}, \ldots, x_{n}\right]$ can be computed. The only difference is when one has to handle two incomparable (under division) elements $a, b$ in $\mathbf{R}$. In this situation, one should first compute $u, v, w \in \mathbf{R}$ such that

$$
\left\{\begin{array}{l}
u b=v a \\
w b=(1-u) a .
\end{array}\right.
$$

Henceforth, one opens two branches: the computations are pursued in $\mathbf{R}_{u}$ and $\mathbf{R}_{1+u \mathbf{R}}:=\{(x / y), x \in \mathbf{R}$ and $\exists z \in \mathbf{R}$ such that $y=1+z u\}$. Note that contrary to [11], the localization $\mathbf{R}_{1+u \mathbf{R}}$ instead of $\mathbf{R}_{1-u}$ is used in order to avoid redundancies. The Dedekind $\operatorname{ring} \mathbf{R}$ is forced to behave like a valuation ring and this situation will produce a binary tree in which leaves correspond to localizations $S_{j}^{-1} \mathbf{R}$, $1 \leq j \leq k$, of $\mathbf{R}$ at comaximal multiplicative subsets $S_{1}, \ldots, S_{k}$. 
The fact that a basis for $\operatorname{Syz}\left(f_{1}, \ldots, f_{s}\right)$ can be computed at each leaf together with Lemma 1 will yield the desired one.

Let $H_{j}=\left\{h_{j, 1}, \ldots, h_{j, p_{j}}\right\}$ denote a basis for $\operatorname{Syz}\left(f_{1}, \ldots, f_{s}\right)$ over $\left(S_{j}^{-1} \mathbf{R}\right)\left[x_{1}, \ldots, x_{n}\right], 1 \leq j \leq k$. There exists a $d_{j} \in S_{j}$ such that $d_{j} h_{j, i} \in \mathbf{R}\left[x_{1}, \ldots, x_{n}\right]$, for each $1 \leq i \leq p_{j}$, and $\left\{d_{j} h_{j, 1}, \ldots, d_{j} h_{j, p_{j}}\right\}$ is a generator for $\operatorname{Syz}\left(f_{1}, \ldots, f_{s}\right)$ over $\left(S_{j}^{-1} \mathbf{R}\right)\left[x_{1}, \ldots, x_{n}\right]$. As explained in Theorem II.3.6 [10], we have the following concrete localglobal principle for coherent modules.

Lemma 1. (syzygy, coherent modules). Let $A$ be a ring, $S_{1}, \ldots, S_{n}$ be comaximal monoids, $M$ be a $A$-module, and $a=\left(a_{1}, \ldots, a_{m}\right) \in M^{m}$.

(1) The syzygy module $N \subseteq A^{m}$ of the vector whose elements $a_{i}$ are seen as vectors in $M$ is finitely generated if and only if each syzygy module $N_{i} \subseteq A_{S_{i}}^{m}$ of the $a_{i}$ 's vector $\left(a_{i}\right.$ are seen as vector in $\left.M\right)$ is finitely generated.

(2) $M$ is coherent if and only if each $M_{S_{i}}$ is coherent.

(3) The ring $A$ is coherent if and only if each $A_{S_{i}}$ is coherent.

Proof.

(1) Let $S$ be a monoid in $A$ and $N^{\prime}$ be the syzygy module of the vector whose elements $a_{i}$ are considered in $M_{S}$. We will prove that $N_{S}=N^{\prime}$. It is clear that $N_{S} \subseteq N^{\prime}$. Conversely, if $\sum_{j=1}^{m}\left(x_{j} / s_{j}\right) a_{j}=0$ in $M_{S}$, let us denote $u=\prod_{i} s_{i}$ and $u_{j}=\prod_{i \neq j} s_{i}$, such that $\sum_{j=1}^{m} x_{j} u_{j} a_{j}=0$ in $M_{S}$ and $\sum_{j=1}^{m} s x_{j} u_{j} a_{j}=0$ in $M$ for $s \in S$. We have $y=\left(y_{1}, \ldots, y_{m}\right)=\left(s x_{1} u_{1}, \ldots\right.$, $\left.s x_{m} u_{m}\right) \in N$ and $\left(\left(x_{1} / s_{1}\right), \ldots,\left(x_{m} / s_{m}\right)\right)=(1 / s u) y$ in $A_{S}$.

(2) Let $a=\left(a_{1}, \ldots, a_{m}\right) \in M^{m}$ and $N \subseteq A^{m}$ be the module of relations for $a$. For all monoids $S, N_{S}$ is the module of relations for $a$ in $M_{S}$.

(3) Is a particular case of 2.

Theorem 1. Let $a=\left(a_{1}, \ldots, a_{m}\right) \in \mathbf{R}\left[x_{1}^{ \pm 1}, \ldots, x_{n}^{ \pm 1}\right]^{m}$ and $\tilde{a}=u\left(a_{1}, \ldots, a_{m}\right)$, where $u=x_{1}^{k_{1}} \ldots x_{n}^{k_{n}}$ is a polynomial in $\mathbf{R}\left[x_{1}, \ldots, x_{n}\right]$ such that $\tilde{a}=\left(\tilde{a}_{1}, \ldots, \tilde{a}_{m}\right) \in \mathbf{R}\left[x_{1}, \ldots, x_{n}\right]^{m}$. The syzygy module $N$ of a over $\mathbf{R}\left[x_{1}^{ \pm 1}, \ldots, x_{n}^{ \pm 1}\right]$ is the same than the syzygy module of $\widetilde{a}$ over $\mathbf{R}\left[x_{1}, \ldots, x_{n}\right]$.

Proof. Let us denote by $N \subseteq \mathbf{R}\left[x_{1}^{ \pm 1}, \ldots, x_{n}^{ \pm 1}\right]^{m}$ the syzygy module of $a$ over $\mathbf{R}\left[x_{1}^{ \pm 1}, \ldots, x_{n}^{ \pm 1}\right]$ and $N^{\prime} \subseteq \mathbf{R}\left[x_{1}, \ldots, x_{n}\right]^{m}$ that of $\tilde{a}$ in $\mathbf{R}\left[x_{1}, \ldots, x_{n}\right]$.

If $\quad \sum_{j=1}^{m} x_{j} \widetilde{a}_{j}=0 \quad$ over $\mathbf{R}\left[x_{1}, \ldots, x_{n}\right], \quad$ then $\sum_{j=1}^{m} x_{j}\left(\tilde{a}_{j} / u\right)=\sum_{j=1}^{m} x_{j} a_{j}=0$, so we have $N \prime \subseteq N$.

Conversely if $\sum_{j=1}^{m} x_{j} a_{j}=0$ over $\mathbf{R}\left[x_{1}^{ \pm 1}, \ldots, x_{n}^{ \pm 1}\right]$, we can suppose that $x_{i} \in \mathbf{R}\left[x_{1}, \ldots, x_{n}\right]^{m}$ (the set of generators of $a$ over $\mathbf{R}\left[x_{1}^{ \pm 1}, \ldots, x_{n}^{ \pm 1}\right]^{m}$ can be supposed with only elements of $\left.\mathbf{R}\left[x_{1}, \ldots, x_{n}\right]\right)$ and we can write $\sum_{j=1}^{m} x_{j} u a_{j}=\sum_{j=1}^{m} x_{j} \widetilde{a}_{j}=0$. Finally, $N=N^{\prime}$.

\section{Syzygies of Laurent Polynomials over Dedekind Ring}

Now, we can give a method for computing a set of generators for the syzygy module, $\operatorname{Syz}\left(f_{1}, \ldots, f_{s}\right)$, over the Laurent polynomial $\operatorname{ring} \mathbf{R}\left[x_{1}^{ \pm 1}, \ldots, x_{n}^{ \pm 1}\right]$. For this, we need the following.

Lemma 2 (see [14]). Let I be the ideal of $\mathbf{R}\left[x_{1}, \ldots, x_{n}, y\right]$ generated by $x_{1} \ldots x_{n} y-1$; then,

$$
\mathbf{R}\left[x_{1}^{ \pm 1}, \ldots, x_{n}^{ \pm 1}\right] \cong \frac{\mathbf{R}\left[x_{1}, \ldots, x_{n}, y\right]}{I} .
$$

Proof. Let $\phi$ be an $\mathbf{R}$-algebra homomorphism defined from $\mathbf{R}\left[x_{1}, \ldots, x_{n}, y\right]$ to $\mathbf{R}\left[x_{1}^{ \pm 1}, \ldots, x_{n}^{ \pm 1}\right]$ by

$$
\begin{aligned}
\phi\left(x_{i}\right) & =x_{i}, \\
\phi(y) & =x_{1}^{-1} \ldots x_{n}^{-1}
\end{aligned}
$$

and extended to $\mathbf{R}\left[x_{1}, \ldots, x_{n}, y\right]$ as follows: for $\alpha \in \mathbf{R}$ and $f, g \in \mathbf{R}\left[x_{1}, \ldots, x_{n}, y\right]$,

$$
\begin{aligned}
\phi(\alpha f) & =\alpha \phi(f), \\
\phi(f+g) & =\phi(f)+\phi(g), \\
\phi(f g) & =\phi(f) \phi(g) .
\end{aligned}
$$

Considering such isomorphism, our problem is reduced to a computation over the polynomial ring $\mathbf{R}\left[x_{1}, \ldots, x_{n}, y\right]$. More precisely, we are going to rely on such isomorphism between $\mathbf{R}\left[x_{1}^{ \pm 1}, \ldots, x_{n}^{ \pm 1}\right]$ and $\left(\mathbf{R}\left[x_{1}, \ldots, x_{n}, y\right] / I\right)$ to obtain an algorithm computing syzygy basis in $\mathbf{R}\left[x_{1}^{ \pm 1}, \ldots, x_{n}^{ \pm 1}\right]$.

The previous isomorphism is well defined, as it does not depend on the way elements of $\mathbf{R}\left[x_{1}, \ldots, x_{n}, y\right] / I$ and $\mathbf{R}\left[x_{1}^{ \pm 1}, \ldots, x_{n}^{ \pm 1}\right]$ are represented. First, every Laurent polynomial can be written as a polynomial in the variables $x_{1}, \ldots, x_{n}, x_{1}^{-1} \ldots x_{n}^{-1}$, and to get an element of $\mathbf{R}\left[x_{1}\right.$, $\left.\ldots, x_{n}, y\right]$ from a Laurent polynomial by this isomorphism, we have to consider the relation $x_{1} \ldots x_{n} y=1$ on $I$.

Note that if $t=x_{1}^{e_{1}} \ldots x_{n}^{e_{n}}$ with $e_{i} \in \mathbb{Z}$ being a term of $\mathbf{R}\left[x_{1}^{ \pm 1}, \ldots, x_{n}^{ \pm 1}\right]$ and $e=-\min \left\{0, e_{1}, \ldots, e_{n}\right\}$, we have

$$
\phi^{-1}(t)=x_{1}^{e_{1}+e} \ldots x_{n}^{e_{n}+e} \cdot y^{e} \in \mathbf{R}\left[x_{1}, \ldots, x_{n}, y\right] \text {. }
$$

Conversely, each element of $\mathbf{R}\left[x_{1}, \ldots, x_{n}, y\right] / I$ is the image of a polynomial in $\mathbf{R}\left[x_{1}, \ldots, x_{n}, y\right]$ through the canonical (surjective) homomorphism of $\mathbf{R}$-algebras:

$$
\mathbf{R}\left[x_{1}, \ldots, x_{n}, y\right] \rightarrow \frac{\mathbf{R}\left[x_{1}, \ldots, x_{n}, y\right]}{I},
$$

which converts $f \in \mathbf{R}\left[x_{1}, \ldots, x_{n}, y\right]$ into $\bar{f}=f(\bmod (I))$.

Given $f \in \mathbf{R}\left[x_{1}, \ldots, x_{n}, y\right]$, we obtain $\bar{f}$ by replacing $x_{i}$ by $\bar{x}_{i}$ and $y$ by $\bar{y}$, bearing in mind that $\bar{x}_{1} \cdots \bar{x}_{n} \cdot \bar{y}=1$. Each $\vec{f} \in \mathbf{R}\left[x_{1}, \ldots, x_{n}, y\right] / I$ can be expressed as algebraic combination of $\bar{x}, \ldots, \bar{x}_{n}, \bar{y}$, with coefficients in $R$. By taking this expression of $\bar{f}$ without the bars over variables, we get a polynomial $p \in \mathbf{R}\left[x_{1}, \ldots, x_{n}, y\right]$ such that $\bar{p}=\bar{f}$. Also, each $g$ with $\bar{f}$ as image can be written as $g=p+\left(x_{1} \ldots x_{n} y-\right.$ 1) $q$ where $q \in \mathbf{R}\left[x_{1}, \ldots, x_{n}, y\right]$. 
By the isomorphism between $\mathbf{R}\left[x_{1}^{ \pm 1}, \ldots, x_{n}^{ \pm 1}\right]$ and $\mathbf{R}\left[x_{1}, \ldots, x_{n}, y\right] / I$, we get the image of $\bar{f}$ replacing $\bar{x}_{i}$ by $x_{i}$ and $\bar{y}$ by $x_{1}^{-1} \cdots x_{n}^{-1}$.

Theorem 2. Let $\left\{s_{1}, \ldots, s_{m}\right\}$ be a set of generators for $\operatorname{Syz}\left(f_{1}, \ldots, f_{r}, x_{1} \ldots x_{n} y-1\right)$; then, $\left\{\bar{s}_{1}, \ldots, \bar{s}_{m}\right\}$ is a set of generators for $\operatorname{Syz}\left(\bar{f}_{1}, \ldots, \bar{f}_{r}\right)$.

Proof. Since $\bar{q}_{1} \bar{f}_{1}+\cdots+\bar{q}_{r} \bar{f}_{r}=0$ is equivalent to $q_{1} f_{1}+$ $\cdots+q_{r} f_{r}+q \cdot\left(x_{1} \ldots x_{n} y-1\right)=0$ with $q \in \mathbf{R}\left[x_{1}, \ldots, x_{n}, y\right]$, the $\mathbf{R}\left[x_{1}, \ldots, x_{n}, y\right]$-homomorphism

$$
\begin{aligned}
& \operatorname{Syz}\left(f_{1}, \ldots, f_{r}, x_{1} \ldots x_{n} y-1\right) \longrightarrow \operatorname{Syz}\left(\bar{f}_{1}, \ldots, \bar{f}_{r}\right), \\
& s=\left(q_{1}, \ldots, q_{r}, q\right) \longmapsto \bar{s}=\left(\bar{q}_{1}, \ldots, \bar{q}_{r}\right)
\end{aligned}
$$

is surjective. We conclude that if $\left\{s_{1}, \ldots, s_{m}\right\}$ is a set of generators for $\operatorname{Syz}\left(f_{1}, \ldots, f_{r}, x_{1} \ldots x_{n} y-1\right)$, then $\left\{\bar{s}_{1}, \ldots, \bar{s}_{m}\right\}$ is a set of generators for $\operatorname{Syz}\left(\bar{f}_{1}, \ldots, \bar{f}_{r}\right)$.

\section{Illustrative Examples}

Example 1. Let $I$ be the ideal of $\mathbb{Z}\left[x^{ \pm 1}, y^{ \pm 1}\right]$ generated by

$$
\begin{aligned}
& f_{1}=10 y^{-1}+1, \\
& f_{2}=6 x^{2} y+3 x^{-1} y^{-1}, \\
& f_{3}=12 x-y+6 x^{-2} y^{-2}-10 .
\end{aligned}
$$

To compute a set of generators for $\operatorname{Syz}\left(f_{1}, f_{2}, f_{3}\right)$, we rely on the isomorphism between $\mathbb{Z}\left[x^{ \pm 1}, y^{ \pm 1}\right]$ and $\mathbb{Z}[x, y, t] /\langle x y t-1\rangle$. It is equivalent to compute a set of generators for $\operatorname{Syz}\left(\bar{g}_{1}, \bar{g}_{2}, \bar{g}_{3}\right)$ with

$$
\begin{aligned}
& \bar{g}_{1}=10 \bar{x} \bar{t}+1, \\
& \bar{g}_{2}=6 \bar{x}^{2} \bar{y}+3 \bar{t}, \\
& \bar{g}_{3}=12 \bar{x}-\bar{y}+6 \bar{t}^{2}-10 .
\end{aligned}
$$

First of all, let us compute in $\mathbb{Z}[x, y, t]$ a set of generators for $\operatorname{Syz}\left(g_{1}, g_{2}, g_{3}, g_{4}\right)$ with

$$
\begin{aligned}
& g_{1}=10 x t+1, \\
& g_{2}=6 x^{2} y+3 t, \\
& g_{3}=12 x-y+6 t^{2}-10, \\
& g_{4}=x y t-1 .
\end{aligned}
$$

Let us use the lexicographic order with $x>t>y$ as monomial order to compute a dynamical Gröbner basis for $J=$ $\left\langle g_{1}, g_{2}, g_{3}, g_{4}\right\rangle$ in $\mathbb{Z}[x, y, t]$. As the leading coefficients of $g_{1}$ and $g_{2}$ are not comparable under division in $\mathbb{Z}$ and $\operatorname{lcm}(10,6)=2$, we can open two following leaves to proceed:

$$
\underset{\mathbb{Z}_{5}}{\swarrow} \mathbb{Z}_{3}^{\mathbb{Z}}
$$

In $\mathbb{Z}_{5}, \quad S\left(g_{1}, g_{2}\right)=(3 / 5) x y g_{1}-t g_{2}=(3 / 5) x y-$ $3 t^{2}:=g_{5}$. Since the leading coefficients of $g_{1}$ and $g_{5}$ are not comparable, we need to open two new leaves as

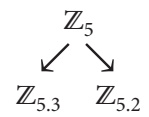

In $\mathbb{Z}_{5.3}, G_{5.3}=\left\{y+10,2 x+t^{2}, 1-5 t^{3}\right\}$ is a special Gröbner basis for $J$ at the leaf $\mathbb{Z}_{5.3}$. And over $\mathbb{Z}_{5.3}[x, y, t]$, we have $\operatorname{Syz}\left(g_{1}, g_{2}, g_{3}, g_{4}\right)$ as

$$
\begin{aligned}
& \mid\left(\begin{array}{c}
2 x y+y t^{2} \\
\frac{t y+10 t}{3} \\
0 \\
-10 t^{2}+2 x y
\end{array}\right),\left(\begin{array}{c}
y t^{3}+2 \\
\frac{t^{2} y+10 t^{2}}{3} \\
-10 t^{3}+2+2 x y t+20 x t
\end{array}\right),\left(\begin{array}{c}
\frac{2 x+t^{2}}{5} \\
0 \\
\frac{2}{5} x+4 x^{2} t
\end{array}\right) \\
& \left(\begin{array}{c}
3 x y t^{2}-3 t \\
-x y t+1 \\
0 \\
-2 t \\
-30 x t^{2}+6 x^{2} y
\end{array}\right),\left(\begin{array}{c}
\frac{1}{15} t-\frac{2}{3} x t^{2} \\
1 \\
0 \\
0 \\
-10+12 x
\end{array}\right),
\end{aligned}
$$

In $\mathbb{Z}_{5.2}$, we find $G_{5.2}=\left\{y+10,6 x+3 t^{2},(3 / 5)\right.$ $\left.-3 t^{3}, 10 x t+1\right\}$ as a special Gröbner basis for $J$ at the leaf
$\mathbb{Z}_{5.2}$, and we have the following $\operatorname{Syz}\left(g_{1}, g_{2}, g_{3}, g_{4}\right)$ over $\mathbb{Z}_{5.2}[x, y, t]:$ 


$$
\begin{aligned}
& \left(\begin{array}{c}
-\frac{3}{10} t^{2}-\frac{3}{5} x \\
\frac{1}{10} t+10 x t^{2} \\
0 \\
-\frac{3}{5} x-6 x^{2} t
\end{array}\right),\left(\begin{array}{c}
6 x y+3 y t^{2} \\
-t y-10 t \\
0 \\
\frac{6}{5} x+\frac{3}{5} t^{2} \\
-30 t^{2}+6 x y
\end{array}\right),\left(\begin{array}{c}
-6-3 y t^{3} \\
t^{2} y+10 t^{2} \\
0 \\
-\frac{1}{5} t-2 x t^{2} \\
0 \\
\frac{6}{5} x+12 x^{2} t
\end{array}\right),\left(\begin{array}{c}
3 x y t^{2}-3 t \\
-x y t+1 \\
0 \\
y \\
-30 x t^{2}+6 x^{2} y
\end{array}\right),\left(\begin{array}{c}
-2 t \\
1 \\
-10+12 x
\end{array}\right),\left(\begin{array}{c}
-x y t+1 \\
0 \\
0 \\
0 \\
10 x t+1
\end{array}\right) \\
& \left(\begin{array}{c}
0 x t \\
\end{array}\right)
\end{aligned}
$$

In $\mathbb{Z}_{3}$, we proceed as above, and we will open two leaves:

$$
\underset{\mathbb{Z}_{3.2}}{\swarrow \mathbb{Z}_{3.5}}
$$

$G_{3.2}=\left\{y+10,2 x+t^{2}, 1-5 t^{3}\right\}$ is a special Gröbner basis for $J$ at the leaf $\mathbb{Z}_{3.2}$, and we get $\operatorname{Syz}\left(g_{1}, g_{2}, g_{3}, g_{4}\right)$ as

$$
\begin{aligned}
& \left\langle\left(\begin{array}{c}
x y+\frac{1}{2} t^{2} \\
\frac{-t y-10 t}{6} \\
0 \\
-5 t^{2}+x y
\end{array}\right),\left(\begin{array}{c}
-5 y t^{3}+10 \\
\frac{5 t^{2} y+50 t^{2}}{3} \\
50 t^{3}-10-10 x y t-100 x t
\end{array}\right)\left(\begin{array}{c}
-x-\frac{1}{2} t^{2} \\
0 \\
\frac{1}{6} t+\frac{5}{3} x t^{2} \\
0 \\
-x y t^{2}-3 t \\
-x y t+1 \\
0 \\
-30 x t^{2}+6 x^{2} y
\end{array}\right),\left(\begin{array}{c}
-2 t \\
1 \\
-10+12 x
\end{array}\right),\left(\begin{array}{c}
-x y t+1 \\
0 \\
0 \\
10 x t+1
\end{array}\right)\right| \\
& \left(\begin{array}{c}
0 \\
0
\end{array}\right)
\end{aligned}
$$

Note that $G_{3.5}=G_{5.3}$.

Finally, $\operatorname{Syz}\left(g_{1}, g_{2}, g_{3}, g_{4}\right)$ over $\mathbb{Z}[x, y, t]$ is 


$$
\begin{aligned}
& \mid\left(\begin{array}{c}
15\left(y t^{3}+2\right) \\
-5 t^{2}(y+10) \\
0 \\
-10 t^{3}+2+2 x y t+20 x t
\end{array}\right),\left(\begin{array}{c}
3 y\left(2 x+t^{2}\right) \\
-t(y+10) \\
0 \\
-30 t^{2}+6 x y
\end{array}\right),\left(\begin{array}{c}
-6 x+3 t^{2} \\
t(1+10 x t) \\
0 \\
-6 x(1+10 x t)
\end{array}\right) \\
& \left(\begin{array}{c}
y \\
-2 t \\
1 \\
-10+12 x
\end{array}\right),\left(\begin{array}{c}
3 x y t^{2}-3 t \\
-x y t+1 \\
0 \\
-30 x t^{2}+6 x^{2} y
\end{array}\right),\left(\begin{array}{c}
-x y t+1 \\
0 \\
0 \\
10 x t+1
\end{array}\right)
\end{aligned}
$$

Note that $\bar{x} \bar{y} \bar{t}=1$. Hence, a set of generators for $\operatorname{Syz}\left(\bar{g}_{1}, \bar{g}_{2}, \bar{g}_{3}\right)$ is

$$
\left\{\left(\begin{array}{c}
15\left(\bar{y} \bar{t}^{3}+2\right) \\
-5 \bar{t}^{2}(\bar{y}+10) \\
0
\end{array}\right),\left(\begin{array}{c}
3 \bar{y}\left(2 \bar{x}+\bar{t}^{2}\right) \\
-\bar{t}(\bar{y}+10) \\
0
\end{array}\right),\left(\begin{array}{c}
-6 \bar{x}+3 \bar{t}^{2} \\
\bar{t}(1+10 \bar{x} \bar{t}) \\
0
\end{array}\right),\left(\begin{array}{c}
\bar{y} \\
-2 \bar{t} \\
1
\end{array}\right)\right\}
$$

Therefore, a set of generators for $\operatorname{Syz}\left(f_{1}, f_{2}, f_{3}\right)$ is

$$
\left\{\left(\begin{array}{c}
15 y^{-2} x^{-3}+30 \\
-5 x^{-2} y^{-1}-50 x^{-2} y^{-2} \\
0
\end{array}\right),\left(\begin{array}{c}
6 x y+3 x^{-2} y^{-1} \\
-x^{-1}-10 x^{-1} y^{-1} \\
0
\end{array}\right),\left(\begin{array}{c}
-6 x-3 x^{-2} y^{-2} \\
x^{-1} y^{-1}+10 x^{-1} y^{-2} \\
0
\end{array}\right),\left(\begin{array}{c}
y \\
-2 x^{-1} y^{-1} \\
1
\end{array}\right)\right\}
$$

Finally, a set of generators of $\operatorname{Syz}\left(f_{1}, f_{2}, f_{3}\right)$ over $\mathbb{Z}\left[x^{ \pm 1}, y^{ \pm 1}\right]$ is

$$
\left\{\left(\begin{array}{c}
6 x y+3 x^{-2} y^{-1} \\
-x^{-1}-10 x^{-1} y^{-1} \\
0
\end{array}\right),\left(\begin{array}{c}
y \\
-2 x^{-1} y^{-1} \\
1
\end{array}\right)\right\}
$$

Now, let us see an example where the basic ring is not principal.

Example 2. Let us consider the ring $\mathbb{Z}[\theta]\left[y^{ \pm 1}, z^{ \pm 1}\right]$, where $\theta^{2}=-5$ is its ideal $I$ generated by

$$
\begin{aligned}
& f_{1}=(8+4 \theta) z^{-1}-y^{-1}+15 y^{-1} z^{-1}, \\
& f_{2}=1+3 z^{-1} \\
& f_{3}=(4+2 \theta) y+9 .
\end{aligned}
$$

To compute a set of generators for $\operatorname{Syz}\left(f_{1}, f_{2}, f_{3}\right)$, we rely on the isomorphism between $\mathbb{Z}[\theta]\left[y^{ \pm 1}, z^{ \pm 1}\right]$ and $\mathbb{Z}[\theta][x, y, z] /\langle x y z-1\rangle$. It is equivalent to compute a set of generators for $\operatorname{Syz}\left(\bar{g}_{1}, \bar{g}_{2}, \bar{g}_{3}\right)$ with

$$
\begin{aligned}
& \bar{g}_{1}=(8+4 \theta) \bar{x} \bar{y}-\bar{x} \bar{z}+15 \bar{x}, \\
& \bar{g}_{2}=3 \bar{x} \bar{y}+1, \\
& \bar{g}_{3}=(4+2 \theta) \bar{y}+9 .
\end{aligned}
$$

First of all, let us compute in $\mathbb{Z}[\theta][x, y, z]$ a set of generators for $\operatorname{Syz}\left(g_{1}, g_{2}, g_{3}, g_{4}\right)$ with

$$
\begin{aligned}
& g_{1}=(8+4 \theta) x y-x z+15 x, \\
& g_{2}=3 x y+1, \\
& g_{3}=(4+2 \theta) y+9, \\
& g_{4}=x y z-1 .
\end{aligned}
$$

Using the lexicographic order with $x>y>z$ as monomial order, we proceed as above by computing a dynamical Gröbner basis for $J=\left\langle g_{1}, g_{2}, g_{3}, g_{4}\right\rangle$ in $\mathbb{Z}[x, y, z]$.

Let us denote by $a$ and $b$ the leading coefficients of $g_{2}$ and $g_{3}$, respectively. Since $a:=3$ and $b:=4+2 \theta$ are not comparable, we have to find $u, v, w \in \mathbb{Z}[\theta]$ such that

$$
\left\{\begin{array}{l}
u b=v a, \\
w b=(1-u) a .
\end{array}\right.
$$


With the solution of this system given by In $\mathbb{Z}[\theta]_{4+2 \theta}$ : $u=5+2 \theta, v=6 \theta$, and $w=-3$, we can open two leaves:

$$
\begin{gathered}
\swarrow \quad \searrow \\
\mathbb{Z}[\theta]_{4+2 \theta} \quad \mathbb{Z}[\theta]_{5+2 \theta}
\end{gathered}
$$

$$
\begin{aligned}
& S\left(g_{2}, g_{3}\right)=g_{2}-\frac{3}{4+2 \theta} g_{3}=-\frac{27}{4+2 \theta} x+1:=g_{5}, \\
& S\left(g_{2}, g_{4}\right)=z g_{2}-3 t g_{4}=z+3:=g_{6}, \\
& S\left(g_{2}, g_{5}\right)=-\frac{9}{4+2 \theta} g_{2}-y t g_{5}=-\frac{9}{4+2 \theta}-y \stackrel{g_{3}}{\longrightarrow} 0, \\
& S\left(g_{2}, g_{6}\right)=z g_{2}-3 x y g_{6}=z-9 x y \stackrel{g_{2}}{\longrightarrow} g_{6} \stackrel{g_{6}}{\longrightarrow} 0, g_{1} \stackrel{g_{3}}{\longrightarrow}-x g_{6} \stackrel{g_{6}}{\longrightarrow} 0, \\
& S\left(g_{3}, g_{5}\right)=-\frac{27}{(4+2 \theta)^{2}} x g_{3}-y g_{5}=-\frac{243}{(4+2 \theta)^{2}} x-y=-\frac{1}{4+2 \theta} g_{3}+\frac{9}{4+2 \theta} g_{5} \stackrel{g_{5}}{\longrightarrow} 0, \\
& S\left(g_{3}, g_{6}\right)=z g_{3}-(4+2 \theta) y g_{6}=9 z-3(4+2 \theta) y=-3 g_{3}+9 g_{6} \stackrel{g_{3}, g_{6}}{\longrightarrow} 0, g_{4} \stackrel{g_{6}}{\longrightarrow}-g_{2} \stackrel{g_{2}}{\longrightarrow} 0, \\
& S\left(g_{5}, g_{6}\right)=z g_{5}+\frac{27}{4+2 \theta} x g_{6}=-3 g_{5}+g_{6} \stackrel{g_{5}}{\longrightarrow}, g_{6} 0 .
\end{aligned}
$$

Thus, $\{(4+2 \theta) y+9,(-27 / 4+2 \theta) x+1, z+3\}$ is a special Gröbner basis for $J$ at the leaf $\mathscr{M}(4+2 \theta)^{-1} \mathbb{Z}=\mathbb{Z}_{4+2 \theta}$.
And we have on $\mathbb{Z}_{4+2 \theta}[x, y, z], \operatorname{Syz}\left(g_{1}, g_{2}, g_{3}, g_{4}\right)$ as

$$
\left|\left(\begin{array}{c}
0 \\
-\frac{(4+2 \theta) y+9}{4+2 \theta} \\
\frac{1+3 x y}{4+2 \theta} \\
0
\end{array}\right),\left(\begin{array}{c}
0 \\
-4 y z-2 \theta y z-9 z \\
12 y+6 \theta y+27
\end{array}\right),\left(\begin{array}{c}
0 \\
12+6 \theta+27 x z 4+2 \theta \\
\frac{-3 x z-9 x}{4+2 \theta} \\
\frac{-81 x+12+6 \theta}{4+2 \theta}
\end{array}\right),\left(\begin{array}{c}
1 \\
x z \\
-2 x \\
-3 x
\end{array}\right)\right|
$$

In $\mathbb{Z}[\theta]_{5+2 \theta}$, as 2 and 3 are not comparable under di-

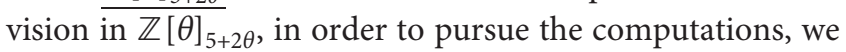
need to open two new leaves:

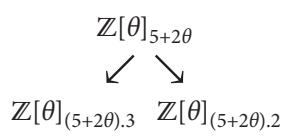

The final evaluation tree is given by

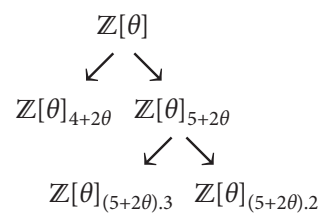

In $\mathbb{Z}[\theta]_{(5+2 \theta) .2}$, we get $\{z+3,(-6+3 \theta / 2) x+1$, $(2 \theta / 5+2 \bar{\theta}) y+1\}$ as a special Gröbner basis for 
$\langle(8+4 a) x y-x z+15 x, 3 x y+1,(4+2 \theta) y+9, x y z-1\rangle, \quad$ at the leaf $\mathscr{M}(5+2 \theta, 2)^{-1} \mathbb{Z}[\theta]=\mathbb{Z}[\theta]_{(5+2 \theta) \cdot 2 .}$. And over $\mathbb{Z}[\theta] \leq{ }_{(5+2 \theta) \cdot 2}[x, y, z], \operatorname{Syz}\left(g_{1}, g_{2}, g_{3}, g_{4}\right)$ is

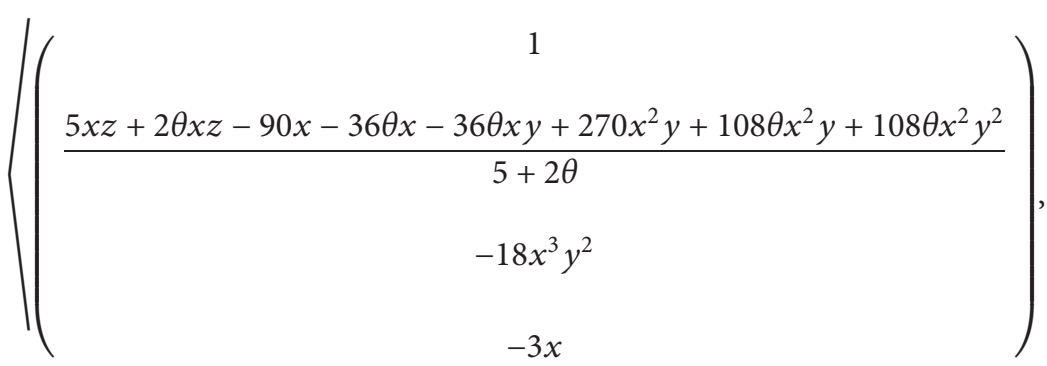

$$
\begin{gathered}
\left(\begin{array}{c}
0 \\
\frac{(2-\theta)(-6 \theta+45 x+18 x \theta+6 x y z \theta+18 x y \theta)}{2(5+2 \theta)} \\
\frac{(\theta-2)\left(x^{2} y z+3 x^{2} y\right)}{2(5+2 \theta)} \\
\frac{(2-\theta)(45 x+18 x \theta-6 \theta)}{2} \\
\frac{(2-\theta)\left(18 \theta x y^{2}+6 x y z \theta-6 \theta y+18 x y \theta+6 \theta x y^{2} z-15+45 x y+15 x y z\right)}{2(5+2 \theta)} \\
\frac{(\theta-2)\left(5 x^{2} y^{2} z+2 \theta x^{2} y^{2} z+15 x^{2} y^{2}+6 \theta x^{2} y^{2}\right)}{2(5+2 \theta)} \\
\frac{(2-\theta)(-6 \theta y-15-6 \theta)}{2(5+2 \theta)}
\end{array}\right),
\end{gathered}
$$

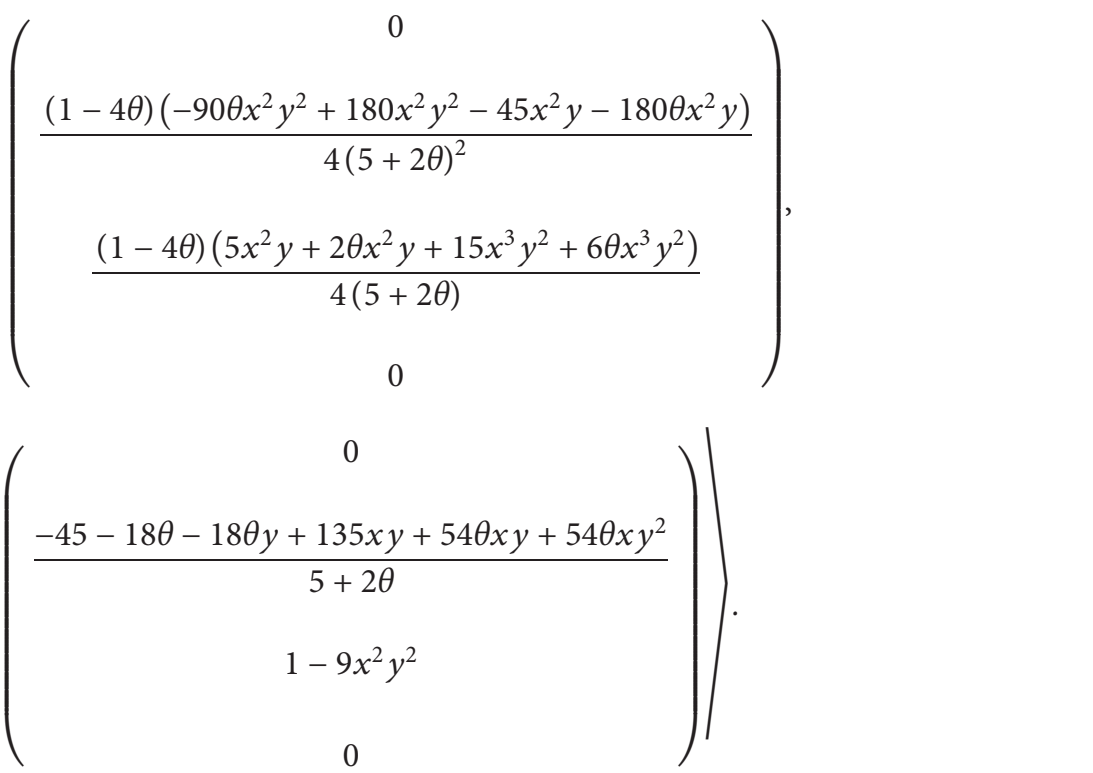


In $\mathbb{Z}[\theta]_{(5+2 \theta) \cdot 3}$, we get $\left\{z+3,3 x-(2 \theta / 5+2 \theta),\left(2 \theta / 5+\quad\right.\right.$ at the leaf $\mathscr{M}(5+2 \theta, 3)^{-1} \mathbb{Z}[\theta]=\mathbb{Z}[\theta](5+2 \theta) .3$. And on $2 \theta) y+1\}$ as a special Gröbner basis for $\mathbb{Z}[\theta]_{(5+2 \theta) \cdot 3}[x, y, z], \operatorname{Syz}\left(g_{1}, g_{2}, g_{3}, g_{4}\right)$ is $\langle(8+4 a) x y-x z+15 x, 3 x y+1,(4+2 \theta) y+9, x y z-1\rangle$,

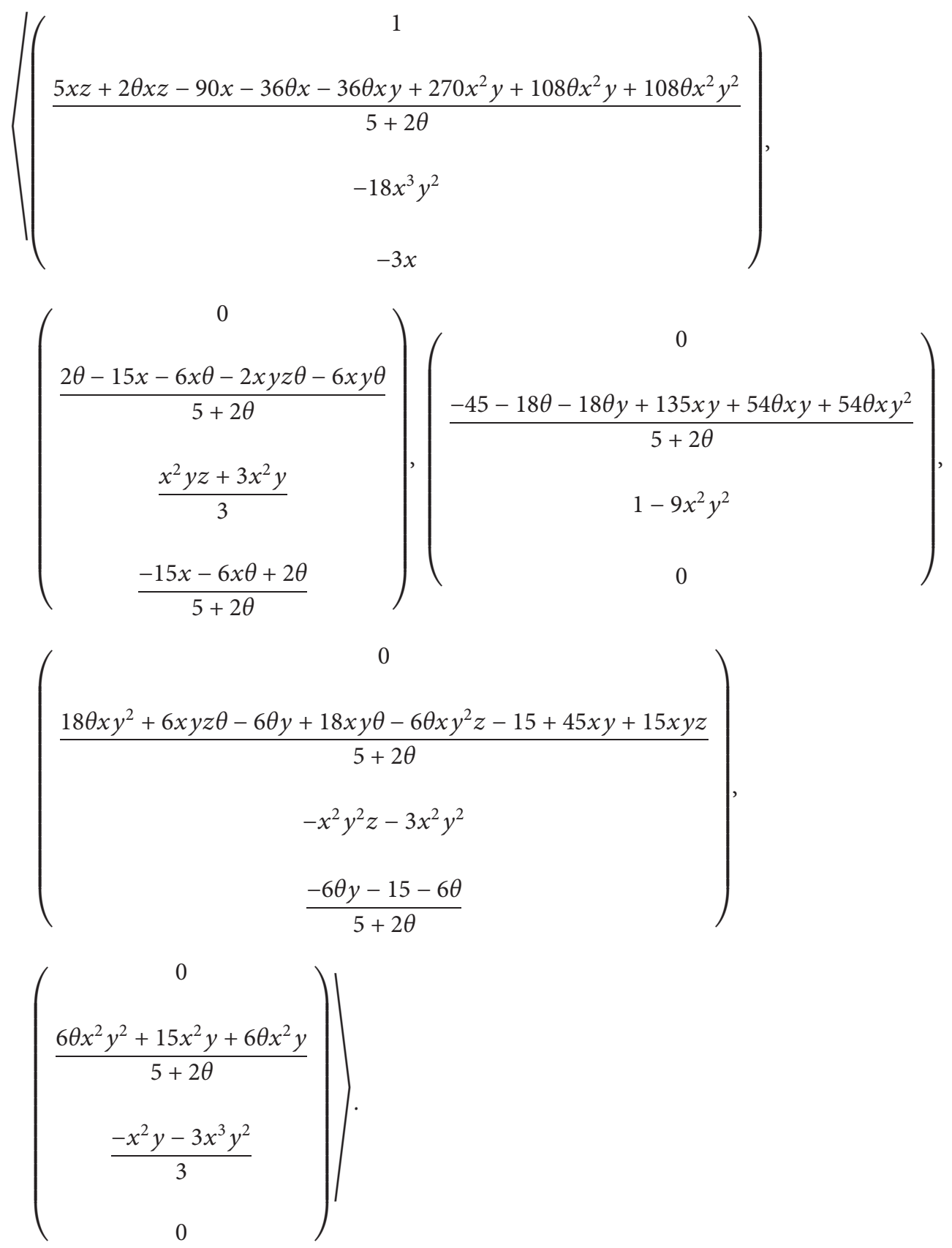

Hence, over $\mathbb{Z}[\theta][x, y, z]$, a generating set of $\operatorname{Syz}\left(g_{1}, g_{2}, g_{3}, g_{4}\right)$ is 


$$
\begin{aligned}
& \left(\left(\begin{array}{c}
0 \\
-4 y-2 \theta y-9 \\
1+3 x y \\
0
\end{array}\right),\left(\begin{array}{c}
0 \\
-4 y z-2 \theta y z-9 z \\
z+3 \\
12 y+6 \theta y+27
\end{array}\right),\left(\begin{array}{c}
1 \\
x z \\
-2 x \\
-3 x
\end{array}\right),\left(\begin{array}{c}
0 \\
12+6 \theta+27 x z \\
-3 x z-9 x \\
-81 x+12+6 \theta
\end{array}\right),\right. \\
& \left(\begin{array}{c}
5+2 \theta \\
5 x z+2 \theta x z-90 x-36 \theta x-36 \theta x y+270 x^{2} y+108 \theta x^{2} y+108 \theta x^{2} y^{2} \\
-(90+36 \theta) x^{3} y^{2} \\
-(15+6 \theta) x
\end{array}\right), \\
& \left(\begin{array}{c}
0 \\
-6 \theta+45 x+18 x \theta(1+y)+6 x y z \theta \\
-(5+2 \theta)\left(x^{2} y z+3 x^{2} y\right) \\
45 x+18 x \theta-6 \theta
\end{array}\right) \\
& \left(\begin{array}{c}
0 \\
18 \theta x y^{2}+6 x y z \theta-6 \theta-6 \theta y+18 x y \theta+6 \theta x y^{2} z-15+45 x y+15 x y z \\
-5 x^{2} y^{2} z-2 \theta x^{2} y^{2} z-15 x^{2} y^{2}-6 \theta x^{2} y^{2} \\
-6 \theta y-15-6 \theta
\end{array}\right) \text {, } \\
& \left(\begin{array}{c}
0 \\
-90 \theta x^{2} y^{2}+180 x^{2} y^{2}-45 x^{2} y-180 \theta x^{2} y \\
(5+2 \theta)\left(5 x^{2} y+2 \theta x^{2} y+15 x^{3} y^{2}+6 \theta x^{3} y^{2}\right) \\
0
\end{array}\right)
\end{aligned}
$$

Note that we have $\bar{x} \bar{y} \bar{z}=1$, and over $\mathbb{Z}[\theta]\left[y^{ \pm 1}, z^{ \pm 1}\right]$, the generation is reduced to $\operatorname{Syz}\left(f_{1}, f_{2}, f_{3}\right)$ as

$$
\left\lfloor\left(\begin{array}{c}
1 \\
\frac{1}{y} \\
z+3 \\
-\frac{2}{y z}
\end{array}\right),\left(\begin{array}{c}
5+2 \theta \\
-4 y z-2 \theta y z-9 z \\
z
\end{array}\right),\left(\begin{array}{c}
\frac{5+2 \theta}{y}-\frac{90+36 \theta}{y z}-\frac{36 \theta}{z}+\frac{270+108 \theta}{y z^{2}}+\frac{108 \theta}{y^{2} z^{2}} \\
\frac{-90-36 \theta}{y z^{3}}
\end{array}\right) \mid .\right.
$$

In fact the trick in Lemma 2 can be used for computing syzygies of a finite system in any module $M[1 / u]$ over $A[1 / u]$ when $A$ is a coherent $A$-module. Here, $M=A=$ $\mathbf{R}\left[x_{1}, \ldots, x_{n}\right]$ and $u=x_{1} \ldots x_{n}$.

Note that a generator set for the syzygy module of $\left(f_{1}, \ldots, f_{s}\right)$ over the Laurent polynomial ring $\mathbf{R}\left[x_{1}^{ \pm 1}, \ldots, x_{n}^{ \pm 1}\right]$ can be directly computed in $\mathbf{R}\left[x_{1}, \ldots, x_{n}\right]$ by multiplying $\left(f_{1}, \ldots, f_{s}\right)$ by a polynomial such that the new vector obtained is in $\mathbf{R}\left[x_{1}, \ldots, x_{n}\right]$, by virtue of Theorem 1 . The syzygy module does not change and the problem is reduced to a computation of a generator set for the syzygy module in $\mathbf{R}\left[x_{1}, \ldots, x_{n}\right]$.
Example 3. Let $I=\left\langle F_{1}=8 Y^{-1}+X^{-2} Y^{-2}, F_{2}=10 X Y^{-2}\right.$ $\left.-2 X^{-2} Y^{-2}\right\rangle$ in $\mathbb{Z}\left[X^{ \pm 1}, Y^{ \pm 1}\right]$. The problem of computing a generator for the syzygy module of $I$ can be reduced to find a generator for the syzygy module of $\left\langle f_{1}=8 X^{2} Y+1, f_{2}=10 X^{3}-2\right\rangle$ in $\mathbb{Z}[X, Y]$.

$\left(f_{1}, f_{2}\right)$ is obtained by multiplying $\left(F_{1}, F_{2}\right)$ by $X^{2} Y^{2}$. Let us consider the lexicographic order with $X>Y$.

As $8 \wedge 10=2,8=2 \times 4$, and $10=2 \times 5$, we have to open two leaves: $\mathbb{Z}_{4}$ and $\mathbb{Z}_{5}$.

In $\mathbb{Z}_{4}, S\left(f_{1}, f_{2}\right)=(5 / 4) X f_{1}-Y f_{2}=(5 / 4) X+2 Y=:$ $f_{3}$. The leading coefficients of $f_{1}$ and $f_{3}$ are comparable 
under division. As $8 \wedge(5 / 4)=2 \wedge 5=1$, we open in $\mathbb{Z}_{4}$ two $\quad$ In $\mathbb{Z}_{4.2}$ : new leaves $\mathbb{Z}_{4.2}$ and $\mathbb{Z}_{4.5}$.

$$
\begin{aligned}
& S\left(f_{1}, f_{3}\right)=(5 / 32) f_{1}-X Y f_{3}=\left(\frac{5}{32}\right)-2 X Y^{2}:=f_{4}, \\
& S\left(f_{1}, f_{4}\right)=Y f_{1}+4 X f_{4}=\left(\frac{1}{2}\right) f_{3} \stackrel{f_{3}}{\longrightarrow} 0, \\
& S\left(f_{2}, f_{4}\right)=Y^{2} f_{2}+5 X^{2} f_{4}=\left(\left(\frac{5}{8}\right) X-Y\right) f_{3} \stackrel{f_{3}}{\longrightarrow} 0, \\
& S\left(f_{3}, f_{4}\right)=Y^{2} f_{3}+(5 / 8) f_{4}=2 Y^{3}+\left(\frac{25}{256}\right):=f_{5}, \\
& S\left(f_{1}, f_{5}\right)=Y^{2} f_{1}-4 X^{2} f_{5}=\left(-\left(\frac{5}{16}\right) X+\left(\frac{1}{2}\right) Y\right) f_{3} \stackrel{f_{3}}{\longrightarrow} 0, \\
& S\left(f_{2}, f_{5}\right)=Y^{3} f_{2}-5 X^{3} f_{5}=\left(-\left(\frac{25}{64}\right) X^{2}+\left(\frac{5}{8}\right) X Y-Y^{2}\right) f_{3} \stackrel{f_{3}}{\longrightarrow} 0 \ldots, \\
& S\left(f_{3}, f_{5}\right)=Y^{3} f_{3}-\left(\frac{5}{8}\right) X f_{5} \stackrel{f_{3}}{\longrightarrow} Y f_{5} \stackrel{f_{5}}{\longrightarrow} 0, S\left(f_{4}, f_{5}\right)=Y f_{4}+X f_{5}=\left(\frac{5}{64}\right) f_{3} \stackrel{f_{3}}{\longrightarrow} 0 .
\end{aligned}
$$

So, $G_{1}=\left\{f_{1}, f_{2}, f_{3}, f_{4}, f_{5}\right\}$ is a special Gröbner basis for

We obtain $\left\langle f_{1}, f_{2}\right\rangle$ over $\mathbb{Z}_{4.2}[X, Y]$.

$$
\operatorname{Syz}(F)=\left\langle\left(\begin{array}{c}
2-10 X^{3} \\
1+8 X^{2}
\end{array}\right),\left(\begin{array}{c}
Y^{2}-\frac{5}{8} X Y-5 X^{3} Y^{2}+\frac{25}{8} X^{4} Y \\
\frac{1}{2} Y^{2}-\frac{5}{16} X Y+4 X^{2} Y^{3}-\frac{5}{2} X^{3} Y^{2}
\end{array}\right)\right), \text { over } \mathbb{Z}_{4.2}[X, Y]
$$

In $\mathbb{Z}_{4.5}[X, Y]$, we obtain $G_{2}=\left\{1+8 X^{2}, 10 X^{3}-2,(5 / 4)\right.$ $\left.X+2 Y, 1-(64 / 5) X Y^{2}, 2 Y^{3}+(25 / 256)\right\}$ as special Gröbner

Also, basis for $\left\langle f_{1}, f_{2}\right\rangle$ over $\mathbb{Z}_{4.5}[X, Y]$.

$$
\operatorname{Syz}(F)=\left\langle\left(\begin{array}{c}
2-10 X^{3} \\
1+8 X^{2} Y
\end{array}\right),\left(\begin{array}{c}
Y^{2}-\frac{5}{8} X Y-5 X^{3} Y^{2}+\frac{25}{8} X^{4} Y \\
-\frac{5}{16} X Y+\frac{1}{2} Y^{2}+4 X^{2} Y^{3}-\frac{5}{2} X^{3} Y^{2}
\end{array}\right)\right\rangle \text { over } \mathbb{Z}_{4.5}[X, Y]
$$

We find $G_{3}=\left\{1+8 X^{2}, 10 X^{3}-2, X+(8 / 5) Y, 1-\right.$ $\left.(64 / 5) X Y^{2},-(512 / 25) Y^{3}-1\right\}$ as special Gröbner basis for

Also, $\left\langle f_{1}, f_{2}\right\rangle$ over $\mathbb{Z}_{5}[X, Y]$.

$$
\operatorname{Syz}(F)=\left\langle\left(\begin{array}{c}
2-10 X^{3} \\
1+8 X^{2} Y
\end{array}\right),\left(\begin{array}{c}
\frac{8}{5} X Y-\frac{64}{25} Y^{2}-8 X^{4} Y+\frac{64}{5} X^{3} Y 2 \\
\frac{4}{5} X Y-\frac{32}{25} Y^{2}-\frac{256}{25} X^{2} Y^{3}+\frac{32}{5} X^{3} Y^{2}
\end{array}\right)\right\rangle \text { over } \mathbb{Z}_{5}[X, Y] .
$$


Finally, we obtain

$$
\operatorname{Syz}\left(f_{1}, f_{2}\right)=\left\langle\left(\begin{array}{c}
2-10 X^{3} \\
1+8 X^{2} Y
\end{array}\right),\left(\begin{array}{c}
16 Y^{2}-10 X Y-80 X^{3} Y^{2}+50 X^{4} Y \\
-5 X Y+8 Y^{2}+64 X^{2} Y^{3}-40 X^{3} Y^{2}
\end{array}\right)\right\rangle \text {, over } \mathbb{Z}[X, Y]
$$

The two approaches used give similar results. In the approach used in the last example, there is one less polynomial than using the isomorphism approach, but the calculations remain similar and relation $\overline{x_{1}} \ldots \overline{x_{n}}=1$ allows simplifications with the isomorphism approach.

\section{Data Availability}

The data used to support the findings are included within the article and are cited as references.

\section{Conflicts of Interest}

The authors declare that they have no conflicts of interest.

\section{References}

[1] M. Amidou and I. Yengui, "An algorithm for unimodular completion over Laurent polynomial rings," Linear Algebra and Its Applications, vol. 429, no. 7, pp. 1687-1698, 2008.

[2] T. Coquand and H. Lombardi, "Hidden constructions in abstract algebra (3) Krull dimension of distributive lattices and commutative rings," in Commutative Ring Theory and Applications-Lecture Notes in Pure and Applied Mathematics, M. Fontana, S.-E. Kabbaj, S. Wiegand, and M. Dekker, Eds., vol. 131, pp. 477-499, Springer, Berlin, Germany, 2002.

[3] M. Coste, H. Lombardi, and M.-F. Roy, "Dynamical method in algebra: effective nullstellensätze," Annals of Pure and Applied Logic, vol. 111, no. 3, pp. 203-256, 2001.

[4] L. Ducos, H. Lombardi, C. Quitté, and M. Salou, "Théorie algorithmique des anneaux arithmétiques, des anneaux de prüfer et des anneaux de dedekind," Journal of Algebra, vol. 281, no. 2, pp. 604-650, 2004.

[5] M. Gamanda, H. Lombardi, S. Neuwirth, and I. Yengui, "The syzygy theorem for Bézout rings," Mathematics of Computation, American Mathematical Society, vol. 89, pp. 941-964, 2020.

[6] A. Hadj Kacem and I. Yengui, "Dynamical gröbner bases over dedekind rings," Journal of Algebra, vol. 324, no. 1, pp. 12-24, 2010.

[7] H. Lombardi, "Hidden constructions in abstract algebra (1): integral dependance," Journal of Pure and Applied Algebra, vol. 167, no. 2-3, pp. 259-267, 2002.

[8] H. Lombardi, "Constructions cachées en algèbre abstraite (5) Principe local-global de Pfister et variantes," International Journal of Commutative Rings, vol. 2, no. 4, pp. 157-176, 2003.

[9] H. Lombardi and C. Quitté, "Constructions cachées en algèbre abstraite (2) Le principe local-global," in Dans: Commutative Ring Theory and Applications-Lecture Notes in Pure and Applied Mathematics, M. Fontana, S.-E. Kabbaj, S. Wiegand, and M. Dekker, Eds., vol. 231, pp. 461-476, Springer, Berlin, Germany, 2002.

[10] H. Lombardi and C. Quitté, Commutative Algebra: Constructive Methods. Finite Projective Modules. Algebra and Applications,,vol. 20, Springer, Berlin, Germany, 2015..
[11] I. Yengui, "Dynamical gröbner bases," Journal of Algebra, vol. 301, no. 2, pp. 447-458, 2006.

[12] I. Yengui, "Corrigendum to "Dynamical Gröbner bases" [J. Algebra 301 (2) (2006) 447-458] and to "Dynamical Gröbner bases over Dedekind rings" [J. Algebra 324 (1) (2010) 12-24]," Journal of Algebra, vol. 339, no. 1, pp. 370-375, 2011.

[13] I. Yengui, "Constructive commutative algebra: projective modules over polynomial rings and dynamical Gröbner bases," in Lecture Notes in Mathematics, vol. 2138, Springer, Berlin, Germany, 2015.

[14] P. G. Nicolo, "Algorithms computating Gröbner bases and syzygies in a polynomial and a Laurent polynomial setting," Thesis, Universida Da Padova, Padua, Italy, 2000. 